FORMATION Formation emploi

Revue française de sciences sociales

107 | juillet-septembre 2009

La formation professionnelle en Amérique latine

\title{
Postface
}

Les relations entre formations et emplois : une adéquation improbable

\section{Catherine Agulhon}

\section{CpenEdition}

Journals

Édition électronique

URL : http://journals.openedition.org/formationemploi/2025

DOI : 10.4000/formationemploi.2025

ISSN : 2107-0946

Éditeur

La Documentation française

Édition imprimée

Date de publication : 1 septembre 2009

Pagination : 73-76

ISSN : 0759-6340

Référence électronique

Catherine Agulhon, «Postface », Formation emploi [En ligne], 107 | juillet-septembre 2009, mis en ligne le 01 septembre 2011, consulté le 30 octobre 2020. URL : http://journals.openedition.org/ formationemploi/2025 ; DOI : https://doi.org/10.4000/formationemploi.2025

(c) Tous droits réservés 


\section{POSTFACE}

\section{Les relations entre formations et emplois : une adéquation improbable}

Par Catherine Agulhon*

En France, on ne trouve que peu de textes ou d'analyses sur la situation des relations entre formations et emplois en Amérique du sud, comme l'atteste une rapide revue de la littérature française ou traduite sur le sujet. À l'inverse, les Sud-américains tournent leurs regards vers la France, tant les chercheurs qui y puisent des références théoriques pour une analyse critique que les politiques qui s'en inspirent pour agir sur les systèmes de formation et d'emploi. Ce numéro de Formation Emploi comble partiellement un vide et ouvre sur un débat loin d'être achevé. En effet, quatre textes sur l'Argentine, le Mexique et l'Uruguay ne peuvent circonscrire une question aussi vaste à l'échelle d'un sous-continent composé de pays aux histoires particulières et hétérogènes.

On peut cependant souligner que des deux côtés de l'Atlantique, le chômage et la précarité des emplois ont donné une acuité particulière aux questions de formation. Ce n'est pas pour autant que tous les pays européens ou sud-américains ont adopté des solutions similaires ou équivalentes en termes de systèmes de formation professionnelle ou d'emploi, lesquels sont le produit d'histoires politiques, économiques et sociales situées.

Les textes ici publiés l'ont montré, ces questions de formation sont en prise avec l'insertion des jeunes, l'évolution des marchés du travail et des besoins de qualification difficiles à identifier. Ces questions mettent en jeu et en synergie des acteurs publics et privés, tout comme les systèmes éducatifs et productifs, les uns régulés par les institutions publiques, les autres par les entreprises privées.

En Europe, et en France en particulier, la formation professionnelle s'est construite sur le long terme à la marge du système éducatif. Elle est segmentée selon les acteurs qui l'organisent et les publics qu'elle vise. On n'en finirait pas de décrire toutes les situations

* Catherine Agulhon est maître de conférences, habilitée à diriger des recherches, en sciences de l'éducation à I'université Paris Descartes. Elle fait partie du Laboratoire de sociologie le CERLIS /Centre de recherche sur les liens sociaux) et dirige un master professionnel "Coopération internationale en éducation et formation ».

Elle a notamment publié : C. Agulhon et S. Didou Aupetit (dir.) (2007), Les universités. Quelles réformes pour quelle modernité ? Le cas du Mexique, Paris, Publisud. C. Agulhon et A. Xavier de Brito (dir.) (2009), Les étudiants étrangers à Paris. Entre affiliation et repli, Paris, L'Harmattan. Agulhon C. (2007), « La professionnalisation à l'université, une réponse à la demande sociale ", Recherche et Formation, INRP, $n^{\circ}$ 54. Agulhon C. (2008), "La formation dans la recherche en sciences humaines, une place à conquérir », Éducation permanente, n 177-4. 
possibles et les évolutions diversifiées. On peut malgré tout distinguer formation initiale et continue, sous différents statuts (scolaire, salarié, chômeur), formation certifiante ou adaptative, sans prétendre à l'exhaustivité.

De fait, les transformations structurelles de l'économie et des marchés du travail comme la montée du chômage dans le dernier quart du $20^{e}$ siècle ont incité à un renouvellement des politiques de formation et subséquemment, de l'analyse critique de celles-ci par les sociologues et économistes du travail et de l'éducation. On peut identifier quelques moments clés de l'histoire récente de la formation professionnelle qui ont provoqué des renouvellements successifs des analyses théoriques et empiriques des relations entre formations et emplois.

En France, et dans les années 60, c'est la planification nationale qui a initié cette question des relations à construire entre formations et emplois. Elle s'est appuyée sur des exercices de prospective qui partaient d'une vision très mécanique de ces relations qui ont peu à peu été déjouées par les données empiriques (Tanguy, 1986). Dans le même temps, des sociologues, comme B. Charlot, (1979) s'insurgeaient contre l'assujettissement du système éducatif et des jeunes aux besoins de l'économie.

Puis, la décentralisation, dans les années 80, a conduit à reposer ces questions à un niveau infra national. Cette fois encore, les acteurs politiques tentent de mobiliser des éléments de prospective pour adapter finement et localement l'offre de formation aux besoins de l'économie, ce que va une fois encore déjouer l'analyse empirique. En effet, et comme le remarquent ici dans leurs articles, C. Jacinto ou Spinosa et Testa, les besoins de maind'œuvre sont difficilement prédictibles, les temporalités des mondes de l'éducation et du travail ne s'harmonisent pas. L'adéquation formation/emploi est un leurre après lequel courent les acteurs politiques et économiques. Elle peut encore servir de prétexte pour remettre en cause le système éducatif ou tout simplement pour déclasser les jeunes à l'embauche.

Mais la complexité des relations entre formations et emplois tient également à la pluralité des acteurs qu'elles mobilisent, on le voit dans les quatre textes produits ici. S'il est clair que les configurations des systèmes de formation et d'emploi sont très variables d'un pays à l'autre, d'une histoire institutionnelle et économique à l'autre, on remarque que, bien souvent, les ministères de l'Éducation et du Travail, les responsables des régions investissent pour partie la formation, quand les entreprises et les syndicats jouent également un rôle, dans certains types d'actions et de prises de décisions. Trouver un accord entre ces acteurs et un mode de régulation conjoint relève du défi. On peut à la suite d'Annie Vinokur (1995) ou d’Éric Verdier (2008) distinguer des régimes de formation à partir de leurs modes de régulation et des indicateurs qui les caractérisent. Ces régimes englobent des questions idéologiques (principes de justice, conception des individus, modes de classement), des questions institutionnelles et organisationnelles (pilotage, règles de fonctionnement et de financement), ou pédagogiques (conceptions des savoirs et des compétences, modes d'accès et de certification). Ces auteurs distinguent ainsi des régimes: académique (forme scolaire dont les diplômes garantissent un niveau), universitaire (ajoute au modèle précédent une dimension égalitaire), professionnel ou corporatiste (régulé par les organisations professionnelles et les entreprises, vise des métiers, permet des mobilités professionnelles) et marchand (la formation est un produit que l'on vend comme un autre sur un marché concurrentiel).

Ces régimes s'offrent comme une grille d'analyse et permettent de recenser des configurations spécifiques. Ils intègrent plus ou moins les différentes modalités de formation, initiale ou continue, longue ou courte, diplômante ou adaptative et leurs éventuelles articulations.

Dans les articles de ce numéro et en Amérique du Sud, on retrouve cette complexité de systèmes de formation qui s'appuient à la fois sur les ministères de l'Éducation et du Travail, sur les gouvernements régionaux, sur les entreprises, qui visent ici des formations longues inscrites dans le système éducatif (Mexique), là des formations courtes adaptatives financées au niveau fédéral (Uruguay). Les modèles et régimes se télescopent, les initiatives se concurrencent quand la formation censée permettre l'insertion ou le maintien dans l'emploi n'endigue pas ces maux qui passent les frontières: le chômage, le sous- 
emploi et la précarité, le travail informel et sous rémunéré. Comme le soulignent L. Tanguy (1994), ou J. Gautier (1994), la formation, si elle est nécessaire pour faire face aux transformations du travail, ne résout pas les problèmes d'emploi. Elle devient même un substitut à l'emploi dans bien des dispositifs de lutte contre le chômage, une politique sociale (maintenir les individus dans un rapport social) plus qu'une politique d'emploi, une variable d'ajustement structurel qui permet de masquer le chômage.

On le voit, la construction de relations adéquates entre formations et emplois est un vaste chantier toujours en tension. Les gouvernements se succèdent, perpétuent des dispositifs, adoptant une posture plus ou moins libérale, plus ou moins démocratique, sans pour autant garantir la qualification et encore moins l'emploi des travailleurs. Les finalités de la formation sont multiples. Elles peuvent s'ancrer dans la demande sociale, mais aussi entraîner ou dynamiser l'activité économique, ce qui échappe à bien des acteurs politiques ou économiques libéraux qui limitent leur investissement.

Toutefois, on remarquera que l'extension de la problématique à l'enseignement supérieur est à l'ordre du jour en Europe et en France, mais aussi au Mexique ou en Argentine, pour les pays présentés ici. En effet, l'ouverture de l'enseignement supérieur à un plus grand nombre (près de $55 \%$ d'une classe d'âge en France ou 30 \% au Royaume-Uni et moins de $30 \%$ dans la plupart des pays d'Amérique du Sud) s'allie avec une profonde transformation de ses missions. Finaliser les formations, adapter l'offre à la demande sociale, celle des individus, mais aussi celle des entreprises, autant d'actions qui s'inscrivent dans les discours et les réformes comme des finalités premières de l'enseignement supérieur.

$\mathrm{Au}$ Mexique, M. Ibarrola le constate, les jeunes fuient ces formations pragmatiques au profit d'une vision académique du savoir; en Argentine, les écoles techniques n'acquièrent pas le même prestige que l'université traditionnelle. En revanche, en France, les jeunes, surtout dans les milieux populaires, sont en quête de ces formations professionnelles qu'ils jugent efficaces parce qu'assorties d'un stage et d'une ouverture sur le monde du travail auquel ils accèdent toujours plus tard et plus diffi- cilement. Pourtant, ces formations ne sont pas forcément plus opérantes sur le marché du travail.

Les données sur l'insertion des jeunes, en France, produites en partie par le Céreq, ont permis d'établir que moins de $50 \%$ des jeunes, quel que soit leur niveau de formation, accédaient à un emploi en lien avec leur formation (Giret et ali., 2005). M. Ibarrola propose des éléments convergents pour le Mexique. Si le débat idéologique sur la soumission du système éducatif au monde économique est moins exacerbé ces dernières années, les interrogations sur le degré pertinent de spécialisation professionnelle des jeunes dans un monde mouvant sont toujours à l'ordre du jour.

Il n'y a pas, en France, de consensus sur ces questions. Nombre d'acteurs dans les instances de décision, nombre d'enseignants dans les écoles professionnelles sont persuadés que la qualité de l'insertion sociale et professionnelle des jeunes dépend de la qualité de la relation entre la formation et l'emploi. Les débats, les analyses, les faits n’ont pu endiguer une volonté d'adaptation renouvelée de l'offre de formation à l'emploi et même d'une adaptation locale au plus près des entreprises.

En Amérique du Sud, on constate des résistances politiques et organisationnelles tant vis-à-vis de la mise en œuvre d'une politique de formation initiale et continue que de la création des outils d'aide à la décision que sont les enquêtes sur la scolarisation, sur l'insertion des jeunes, sur la mobilité de la population active. En revanche, exhortées par les organisations internationales, ces pays tournent leurs regards vers les expériences européennes, allemandes (système d'apprentissage «dual »), anglaises (polytechnics) ou françaises (dispositifs post scolaires et développement d'une offre de formation initiale, structuration tripartite (État, organisations professionnelles et syndicats) d'un système de formation continue). Ils proposent de la sorte des systèmes multiformes et hybrides où se concurrencent tant les acteurs publics que privés, sans garantir une offre de formation de qualité, susceptible de répondre à la demande sociale. La complexité des interventions et des interactions institutionnelles marque de son empreinte une régulation chaotique de l'offre. 
Enfin, on remarque la porosité d'initiatives européennes quand elles se diffusent largement. En effet, qu'il s'agisse de systèmes de formation ou de modalités spécifiques, on les retrouve dans nombre de pays d’Amérique du Sud. On citera la décentralisation de la définition de l'offre en prise avec le développement local, mais aussi l'idéal de la formation tout au long de la vie (FTLV) avec la diffusion des congés de formation pour les salariés et la création de certifications de branches accréditées soit par les institutions publiques, soit par des agences privées. On citera encore la Validation des acquis de l'expérience (VAE) qui fait lentement son chemin et devrait permettre à des adultes de valider les compétences acquises dans l'expérience. Enfin, la controversée gestion par les compétences incite différents acteurs institutionnels à construire des référentiels de compétences sur lesquels seront forgées des formations segmentées (Monchatre, 2009). Ainsi, une ingénierie de la formation initiée dans maints pays occidentaux (Canada, France...) systématise une approche adéquationniste de ces relations entre formations et emplois que les chercheurs déconstruisent pourtant depuis vingt-cinq ans. Mais l'emprunt dans la construction d'un système trouve ses limites dans la traduction locale qui en est faite. Toute analyse comparative doit tenir compte des contextes spécifiques dans lesquels s'inscrivent ces importations.

Bibliographie

Charlot B. \& M. Figeat (1979), L'école aux enchères, Paris, Éditions Payot.

Gautié J. (1994), « Le chômage des jeunes en France, un problème de formation ? », Futuribles, Avril.

Giret J.-F., Lopez A., Rose J. (2005), Quelles formations pour quels emplois? Paris, La Découverte.

Lefresne F. (2003), Les jeunes et l'emploi, Paris, La Découverte, coll. « Repères ».

Monchatre S. (2009), « Normalisation des compétences et rationalisation pédagogique. Le cas de la formation technique au Québec », Revue du CREN, Recherches en Éducation, ${ }^{\circ} 7$

Tanguy L. et alii (1986), L'introuvable relation formation-emploi, Paris, La Documentation française.
Tanguy L. (2002), « La mise en équivalence de la formation avec l'emploi dans les $\mathrm{IV}^{\circ}$ et $\mathrm{V}^{\circ}$ Plans (1962-1970) », Revue française de sociologie, 43-4.

Tanguy L. (1994), «La formation, une activité sociale en voie de définition ? ", in De Coster M. et Pichault F., Traité de sociologie du travail, Bruxelles, Éditions de Boeck Université.

Verdier E. (2008), « L'éducation et la formation tout au long de la vie : une orientation européenne, des régimes d'action publique et des modèles nationaux en évolution », Sociologie et Sociétés, vol XL, n 1.

Vinokur A. (1995), «Réflexions sur l'économie du diplôme », Formation Emploi, nº 52. 